\title{
An iterative, fast, linear-scaling method for computing induced charges on arbitrary dielectric boundaries
}

Cite as: J. Chem. Phys. 132, 154112 (2010); https://doi.org/10.1063/1.3376011

Submitted: 11 August 2009 . Accepted: 10 March 2010 . Published Online: 21 April 2010

Sandeep Tyagi, Mehmet Süzen, Marcello Sega, Marcia Barbosa, Sofia S. Kantorovich, and Christian Holm

\section{ARTICLES YOU MAY BE INTERESTED IN}

ICMMM2D: An accurate method to include planar dielectric interfaces via image charge summation

The Journal of Chemical Physics 127, 154723 (2007); https://doi.org/10.1063/1.2790428

Electrostatic layer correction with image charges: A linear scaling method to treat slab 2D $+h$ systems with dielectric interfaces

The Journal of Chemical Physics 129, 204102 (2008); https://doi.org/10.1063/1.3021064

Electrochemical interface between an ionic liquid and a model metallic electrode

The Journal of Chemical Physics 126, 084704 (2007); https://doi.org/10.1063/1.2464084

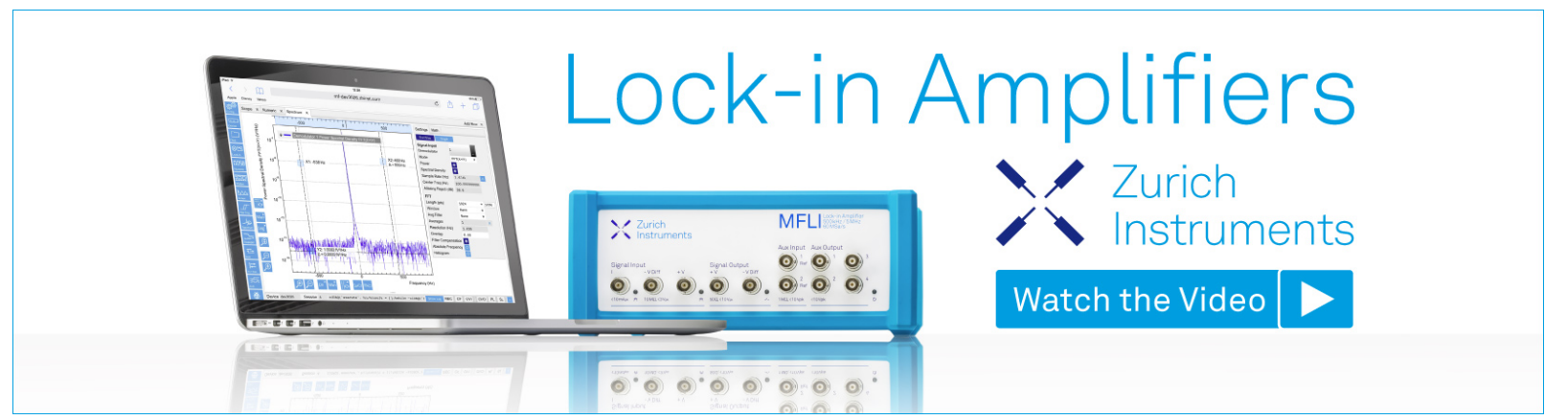

J. Chem. Phys. 132, 154112 (2010); https://doi.org/10.1063/1.3376011

132, 154112

(c) 2010 American Institute of Physics. 


\title{
An iterative, fast, linear-scaling method for computing induced charges on arbitrary dielectric boundaries
}

\author{
Sandeep Tyagi, ${ }^{1}$ Mehmet Süzen, ${ }^{1}$ Marcello Sega,${ }^{2}$ Marcia Barbosa, ${ }^{3}$ \\ Sofia S. Kantorovich, ${ }^{4}$ and Christian Holm ${ }^{4, a)}$ \\ ${ }_{1}^{1}$ Frankfurt Institute for Advanced Studies (FIAS), Ruth-Moufang-Str. 1, 60438 Frankfurt am Main, Germany \\ ${ }^{2}$ Department of Physics, University of Trento and INFN, via Sommarive 14, 38123 Trento, Italy \\ ${ }^{3}$ Instituto de Física, Universidade Federal do Rio Grande do Sul, Porto Alegre, RS 91501-970, Brazil \\ ${ }^{4}$ Institute for Computer Physics, University of Stuttgart, Pfaffenwaldring 27, 70569 Stuttgart, Germany
}

(Received 11 August 2009; accepted 10 March 2010; published online 21 April 2010)

\begin{abstract}
Simulating coarse-grained models of charged soft-condensed matter systems in presence of dielectric discontinuities between different media requires an efficient calculation of polarization effects. This is almost always the case if implicit solvent models are used near interfaces or large macromolecules. We present a fast and accurate method $\left(\mathrm{ICC}^{\star}\right)$ that allows to simulate the presence of an arbitrary number of interfaces of arbitrary shape, each characterized by a different dielectric permittivity in one-, two-, and three-dimensional periodic boundary conditions. The scaling behavior and accuracy of the underlying electrostatic algorithms allow to choose the most appropriate scheme for the system under investigation in terms of precision and computational speed. Due to these characteristics the method is particularly suited to include nonplanar dielectric boundaries in coarse-grained molecular dynamics simulations. () 2010 American Institute of Physics. [doi:10.1063/1.3376011]
\end{abstract}

\section{INTRODUCTION}

Computer simulations are becoming a ubiquitous tool for the investigation of the properties of matter at different time and size scales, and the increasing computational power of modern computers is certainly opening new perspectives, in particular in fields such as soft-condensed matter and biophysics. Nevertheless, the size of target systems and the timescale of the typical relaxation processes are still the limiting factors which preclude the use of models at atomistic detail for many interesting problems. Since such a level of detail is often not required, coarse-graining the uninteresting degrees of freedom is a strategy often employed to simulate larger systems. Needless to say, in many soft-condensed matter systems, the coarse-graining of the degrees of freedom associated to water plays an important role. It often determines which effects can be modeled and up to which length scale the model can be valid. For example, a recent coarsegraining strategy ${ }^{1,2}$ that still represents the solvent explicitly, although removing from it every kind of long-range interaction, has proven to be successful in accounting quantitatively for the hydrophobic effect and reproducing processes such as surfactants aggregation in bilayers. Still, systems in which the electrostatic interaction plays a dominant role (for example, polyelectrolytes and charged hydrogels) could not be modeled accurately in the coarse-grained approach mentioned above. ${ }^{1,2}$ This is not only because of the direct chargecharge interaction at long distances but also because polarization cannot be induced in the model water. There are of course explicit water models which take into account polarizability, see, for example, Refs. 3-5, however, this is done

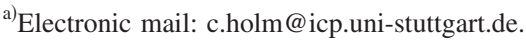

on the atomistic level. At a higher level of coarse-graining one unavoidably reaches the continuum limit. The latter can be refined by using different approaches such as, for example, tailored effective potentials.

Obviously, within the framework of the simplest possible continuum dielectric model where the dielectric permittivity is considered to be homogeneous, not only the entropic effects related to the removed solvent molecules are lost, but also every solvent-related polarization effect other than the general reduction of the Coulomb forces. Many approaches have been developed so far to fill this gap, for example, obtaining the electrostatic energy by solving numerically the Poisson equation (see Refs. 6-9 for instance and references therein). If the ionic atmosphere is also taken into account by using a continuum model, then the Poisson-Boltzmann equation has to be solved (see, for example, Refs. 10-12 and references therein), or, if ionic currents are of interests, the Poisson-Nernst-Planck equation (see, for example, Refs. 13-15 and references therein). Many methods exist to solve these partial differential equations numerically, such as finite differences, finite elements, or boundary element methods.

Boundary element methods are often employed when the solution of the partial differential equations (in the present study we are focusing on the solution of the Poisson equation) is expressed by means of boundary integral equations. Regarding this subject the literature is vast. Here we provide only some ${ }^{11,12,16-21}$ of them, and the reader can refer to review articles if interested in the historical developments in the field. ${ }^{22-24}$ For the case of the Poisson equation with sharp dielectric boundaries, the boundary integral problem consists in determining the induced charge density on the boundary surfaces where the dielectric permittivity has a discontinuity. One of the existing approaches that handles the problem- 
whose most recent formulation is the induced charge computation (ICC) method ${ }^{25}$ - has a long history, dating back most probably to 1966 when Rush and co-workers ${ }^{16}$ first introduced the matrix equation for the induced charges. Later, the same approach has been revived in the context of different applications. ${ }^{17,18,23,26-28}$ The method is based on a discretization of the induced charge density. This discretized density depends linearly on the electrostatic field generated by both real and induced charges resulting in a system of linear equations described by a $M \times M$ matrix elements, where $M$ is the number of surface elements. This system of equations is usually solved by iterative techniques. ${ }^{29}$ In its basic implementation, the ICC method thus scales as $M^{2}$. By recasting the linear system into a self-consistent set of equations it is possible to employ efficient methods for the calculation of the electrostatic interaction (fast multiple method, FMM, for example ${ }^{19}$ ) in order to achieve better scaling. Similar approaches have been employed for the solution of Poissonlike equations such as in Refs. 11, 12, and 30-35.

These works are the starting point of the present paper in which we show that other efficient electrostatics solvers can be used in combination with a self-consistent set of equation for the induced charges in order to obtain a significant speed-up in the computational performances. The target systems for our algorithm (ICC ${ }^{\star}$, where the star stands as a representative for any Poisson solver one likes to employ, since the underlying boundary element method is independent on the periodic boundary conditions as well as the Poisson solver) are, unlike many previously cited works, coarsegrained models in soft-condensed matter. In a typical coarsegraining scheme every charged particle is represented explicitly - be it a macroion, a counterion, or a salt ion-in a medium with homogeneous dielectric permittivity representing a solvent such as water. The boundary surfaces are not meant to mimic molecular surfaces, rather, they represent the effect of static nano- or mesoscopic confining surfaces with mixed dielectric properties. For such systems, periodic boundary conditions are often essential in order to model large ensembles. It should be noted that although the fast multiple method can be used to simulate periodic or partially periodic systems, ${ }^{36,37}$ the electrostatic solvers used in this work can be chosen to optimize the performance of the computation, depending on the characteristics of the physical system under study. Another advantage of the proposed algorithm is the simplicity of its implementation. The method is very general since it determines the dielectric boundary forces without any requirement on the system symmetries for an arbitrary number of generically shaped interfaces of different dielectric permittivity.

In the following section, we will review the theoretical background of $\mathrm{ICC}^{\star}$. In Sec. III we will explain the algorithm, and its results will be checked in Sec. IV against theoretical results for a number of systems for which an exact numerical solution, or alternatively, an appropriately accurate analytical approximation is known. The characteristics and the scaling behavior of the method will be then compared to ICC. Eventually, in Sec. V we draw our conclusions about the methodological advantages and limitations.

\section{THEORY}

We consider a system consisting of a set of point charges (real charges) located at positions $\mathbf{x}_{i}$, which are confined to a region $\mathcal{R}_{1}$ of the space in general disconnected, and characterized by a dielectric permittivity $\epsilon_{1}$. The remaining part $\mathcal{R}_{2}$ of the space which is not accessible by the real charges is assumed to be a medium of permittivity $\epsilon_{2}$. The interface between the two regions will be called $\mathcal{I}$ and has to be smooth (as wedges can introduce a divergent induced charge density). The generalization to regions with different dielectric permittivity is straightforward, providing that each region is characterized by a homogeneous dielectric constant, and there is no contact between more than two different dielectrics. The problem of determining the electrostatic forces on the real charges consists then in solving Poisson equation for the electrostatic potential $\phi$ in the two regions, namely,

$$
\begin{cases}\nabla^{2} \phi=0 & \text { in } \mathcal{R}_{2} \\ \nabla^{2} \phi=4 \pi \sum_{i}^{N} q_{i} \delta\left(\mathbf{r}_{i}-\mathbf{r}\right) / \epsilon_{1} & \text { in } \mathcal{R}_{1},\end{cases}
$$

subject to the requirement of continuity of $\phi$ in the whole space and to the boundary conditions

$$
\epsilon_{1} \mathbf{E}^{(1)} \cdot \mathbf{n}=\epsilon_{2} \mathbf{E}^{(2)} \cdot \mathbf{n},
$$

where $\mathbf{n}$ is the normal vector of the border of $\mathcal{R}_{1}$, by convention pointing into the inner part of region $\mathcal{R}_{2}$. The notation $\mathbf{E}^{(k)}$ indicates that the electric field is evaluated at a position which is arbitrarily close to $\mathcal{I}$ but still belongs to region $\mathcal{R}_{k}$.

The problem of determining the electric field in region $\mathcal{R}_{1}$ can then be simplified employing a physically equivalent system $^{38}$ which satisfies the same Eqs. (1) and (2) in $\mathcal{R}_{1}$. This system consists of a single medium of dielectric constant $\epsilon_{1}$, the original real charges, and a surface charge density $\sigma$ (which will loosely be denoted as induced charge) distributed on $\mathcal{I}$. The continuity requirement and Poisson's equation in region $\mathcal{R}_{1}$ are automatically satisfied in the equivalent problem because no induced charges are placed in region $\mathcal{R}_{1}$ but just on its border $\mathcal{I}$. The boundary condition on $\mathcal{I}$ is now determined by $\sigma$, since the presence of the surface charges introduces a discontinuity in the electric field, such that

$$
\left(\mathbf{E}^{(1)}-\mathbf{E}^{(2)}\right) \cdot \mathbf{n}=4 \pi \sigma / \epsilon_{1} .
$$

A schematic representation of an arbitrarily shaped dielectric interface is shown in Fig. 1. The electrostatic problem becomes then that of finding the distribution $\sigma$ that generates a field which satisfies the boundary conditions (2).

In view of the structure of the $\mathrm{ICC}^{\star}$ method, which is based on a discretization of the dielectric interface surface, from now on we will refer to the discrete analog of the equations for field and charges. Therefore, $\sigma_{i}$ will identify the charge density on the $i$ th discrete surface element of area $a_{i}$ with normal unitary vector $\mathbf{n}_{i}$. The field in the proximity of this surface element in regions $\mathcal{R}_{1}$ and $\mathcal{R}_{2}$ is consistently identified by $\mathbf{E}_{i}^{(1)}$ and $\mathbf{E}_{i}^{(2)}$, respectively. If one now isolates the contribution coming from $\sigma_{i}$ in the expression for the electric field on both sides of $\mathcal{I}$, namely, 


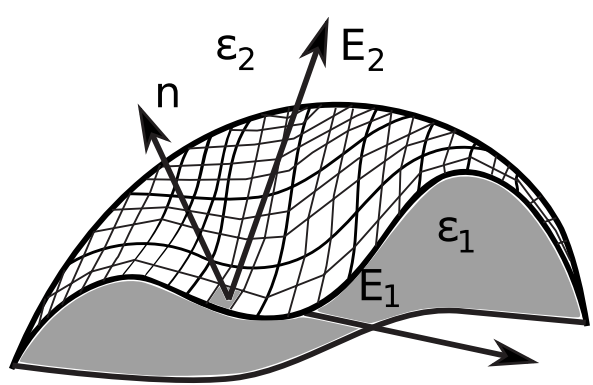

FIG. 1. Sketch of a section of two domains $\mathcal{R}_{1}$ and $\mathcal{R}_{2}$, characterized in the outer and inner regions by the dielectric permittivity $\epsilon_{1}$ and $\epsilon_{2}$, respectively. A discretized surface element is highlighted, and the normal vector $\mathbf{n}$ and the electric fields $\mathbf{E}_{1}$ and $\mathbf{E}_{2}$ at the dividing surface are shown.

$$
\mathbf{E}_{i}^{(1 / 2)}=\mathbf{E}_{i}^{\prime} \pm 2 \pi \sigma_{i} \mathbf{n}_{i} / \epsilon_{1}
$$

it is then possible to obtain a relation between $\sigma_{i}$ and the field $\mathbf{E}_{i}^{\prime}$ generated by all real and induced charges but the $i$ th one. This relation, obtained by combining Eqs. (2)-(4), reads

$$
\sigma_{i}=\frac{\epsilon_{1}}{2 \pi}\left(\frac{\epsilon_{1}-\epsilon_{2}}{\epsilon_{1}+\epsilon_{2}}\right) \mathbf{E}_{i}^{\prime} \cdot \mathbf{n}_{i},
$$

and represents in fact the discretized form of an integral equation for $\sigma_{i}$, since $\mathbf{E}_{i}^{\prime}$ can be expressed as linear combination of all (real and induced) charges, but the induced one located at $\mathbf{x}_{i}$. For convenience we will denote the permittivity-dependent factor in Eq. (5) as

$$
f=\frac{\epsilon_{1}}{2 \pi}\left(\frac{\epsilon_{1}-\epsilon_{2}}{\epsilon_{1}+\epsilon_{2}}\right) .
$$

Equation (5) is the common starting point for induced charge-based methods. ${ }^{19,25,39}$ The strategy which is chosen to solve this equation strongly influences not only the efficiency of the methods, but also their scope, in particular, regarding the type of periodicity which can be treated.

\section{THE ALGORITHM}

Before introducing the $\mathrm{ICC}^{\star}$ algorithm, we will briefly review the basics of a recent similar approach, namely, ICC ${ }^{25,26}$ which we will use as a reference to compare the characteristics and the performances of ICC ${ }^{\star}$. The strategy adopted in the ICC algorithm is to split the electric field $\mathbf{E}_{i}^{\prime}$ into a contribution from the real charges and one from the induced charges $\mathbf{E}_{i}^{\prime}=\mathbf{E}_{i}^{\text {real }}+\mathbf{E}_{i}^{\text {ind }}$. Due to the linearity of Maxwell's equations, the electric field generated by the induced charges can be written as $\mathbf{E}_{i}^{\text {ind }}=\Sigma_{j} K_{i j} \sigma_{j}$, so that Eq. (5) can always be put in the form of a system of linear equations

$$
\sum_{j}\left(\delta_{i j}-f K_{i j}\right) \sigma_{j}=f \mathbf{E}_{i}^{\mathrm{real}} \cdot \mathbf{n}_{i},
$$

where the kernel $K_{i j}$ encodes the geometrical information needed to compute the contribution to the field at position $\mathbf{x}_{i}$ from the induced charge located at $\mathbf{x}_{j}$. The polarization charges in ICC are then determined by solving the linear system

$$
A \mathbf{u}=\mathbf{b},
$$

where $A_{i j}=\left(\delta_{i j}-f K_{i j}\right)$, the components of vector $\mathbf{u}$ are the values of the induced charges $\sigma_{i}$, and the components of $\mathbf{b}$ are $b_{i}=f \mathbf{E}_{i}^{\text {real }} \cdot \mathbf{n}_{i}$. This method is appealing because the matrix inversion has to be performed only at the beginning of the simulation, but it is lacks, at least in the original description of the algorithm, ${ }^{25,26}$ treatment of long-range contributions. (We will turn later on the performances of ICC ${ }^{\star}$ with respect to ICC.)

The ICC ${ }^{\star}$ algorithm basically exploits a different strategy to solve Eq. (5). No matter how the electric field is calculated (for example, using nearest neighbor interaction or taking into account every contribution form periodic images), it can always be expressed as a linear function of the charges in the system, and Eq. (5) is precisely a linear system of equations that is already written in the form which is typical to iterative methods. Our aim is to employ an iterative scheme in the context of periodic systems. Among the many different algorithms ${ }^{40}$ that can be used to implement an iterative solution for Eq. (5), the successive over relaxations (SORs) approach is a very versatile and efficient one. In a SOR scheme, the general equation

$$
\mathbf{u}=B \mathbf{u}+\mathbf{c},
$$

is approximated with desired precision by the sequence of estimates

$$
\mathbf{u}^{(n+1)}=\omega\left(B \mathbf{u}^{(n)}+c\right)+(1-\omega) \mathbf{u}^{(n)} .
$$

Here the superscript $n$ denotes the iteration number and $\omega$ the relaxation parameter, which usually can be in the range $(0,2)$ in order to guarantee convergence. ${ }^{40}$ The iterative solution for the induced charges reads, explicitly,

$$
\sigma_{i}^{(n+1)}=\omega\left(f \mathbf{E}_{i}^{\prime} \cdot \mathbf{n}_{i}\right)+(1-\omega) \sigma_{i}^{(n)} .
$$

The initial values of the induced charges can be extracted form a random distribution. At every iteration the value of the electric field is computed by means of the electrostatics algorithm of choice, and it is used to perform the next SOR approximation of the charge distribution, according to Eq. (11). One usually keeps iterating until a desired accuracy has been achieved. This can be tracked by computing

$$
\Delta=\max _{i=1, M}\left|\frac{\sigma_{i}^{n+1}-\sigma_{i}^{n}}{\sigma_{i}^{n}}\right| .
$$

When $\Delta$ is smaller than some preset tolerance value, the iterative process is stopped. This scheme is highly useful in molecular dynamics (MD) simulations, as it will converge quite fast after the first integration step. Suppose, for example, that for a given configuration of the real charges, the induced ones have been determined up to the desired accuracy. After one MD integration step the induced charge distribution has to be recalculated. However, because the mobile charges normally have moved only slightly from their previous positions, the old induced charge values already serve as good approximation. Therefore, when using these values as a starting guess for the iterative procedure at the next MD step one will obtain a sufficiently well converged solution in, at most, a handful of iterations. In practical ap- 
plications we find that only very few (one to three) iterations are required before the iterative procedure converges again.

Another distinguishing feature of the $\mathrm{ICC}^{\star}$ class of algorithms is that, due to the local formulation of the problem, the generalization to systems with many dielectric interfaces, each having a different dielectric constant, is straightforward. In the general case of many disconnected dielectric regions, the factor $f$ in Eq. (5) will become region-dependent (note, however, that each region has to have a homogeneous dielectric permittivity). Since the iteration procedure is strictly local, i.e., the $f$ factor assigned to each surface element is constant and independent of the other elements, further modifications to the algorithm are not required in order to treat multiple dielectric permittivities.

The last ingredient of the $\mathrm{ICC}^{\star}$ method consists in the way of calculating the electric field $\mathbf{E}_{i}^{\prime}$. The field corresponds to the force acting on the $i$ th particle (generated both by real and induced charges), divided by the charge of the surface element itself. Therefore, the value of the field can be provided by any algorithm which computes the electrostatic force in bulk systems. Fast methods such as FMM, ${ }^{41}$ mesh methods such as P3M (Particle-Particle Particle-Mesh), ${ }^{42-44}$ or methods suitable for periodicity in two or one dimension such as ELC (Electrostatic Layer Correction), ${ }^{45}$ MMM2D ${ }^{46}$ or MMM1D ${ }^{47,48}$ can thus be employed to compute the electric field acting on the boundary elements. This leads to a solution of Eq. (5) that automatically takes into account the periodic boundary conditions of choice. In more detail, P3M is a mesh-based Ewald method that treats the boundary conditions in full three-dimensional (3D) periodicity, and its extension ELC employs analytical corrections in order to deal with 2D periodic boundary conditions. In the bulk, both methods scale like $N \log N$, where $N$ is the number of charges in the system. MMM2D, on the other hand, solves the electrostatics in 2D periodicity exactly. The time needed for MMM2D to compute the forces scales like $N^{5 / 3}$ and, therefore, this method is suitable for relatively small systems only. The MMM2D and ELC algorithms have also variants [ICMMM2D (Ref. 49) and ELCIC (ELC with Image Charges) (Ref. 50)] that can treat planar dielectric discontinuities. Finally, another algorithm of the MMM family that can be used as a solver for bulk electrostatics is MMM1D which handles systems with periodic boundary conditions applied only along one direction. We will make use of the names of these methods to denote the members of the ICC ${ }^{\star}$ class of algorithms that will be therefore called ICCP3M, ICCELC, ICCMMM2D (not to be confused with ELCIC and ICMMM2D), and ICCMMM1D, respectively.

\section{RESULTS}

\section{A. Algorithm validation}

In this section we test the $\mathrm{ICC}^{\star}$ approach for a number of simple geometries. From here on we will use point charges placed at the centers of the surface grid to represent the induced charge distribution. Although the use of a different distribution of charge would lead to higher precision, point charges make the implementation into molecular dynamics code straightforward. Also, in the following we will report

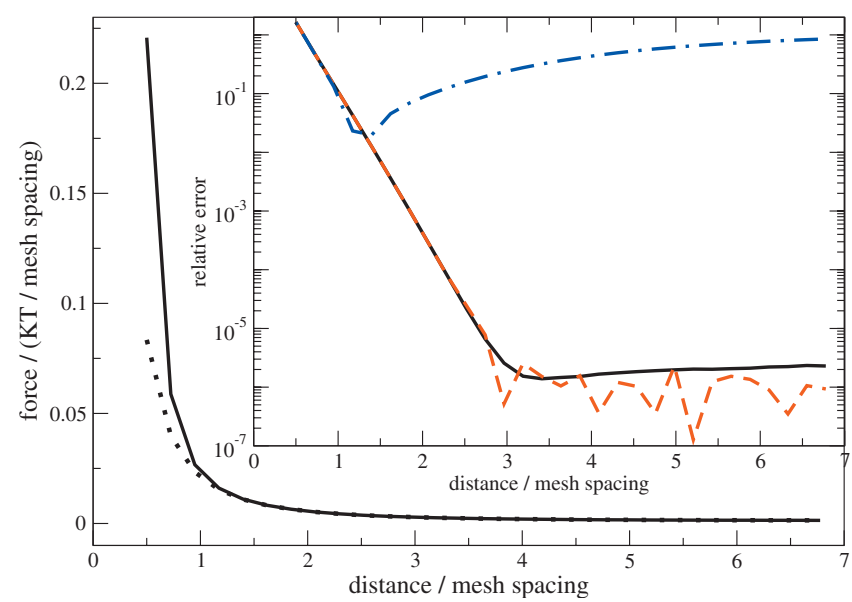

FIG. 2. Force on a single unit charge located at different distances from a dielectric interface $\left(\epsilon_{1}=4, \epsilon_{2}=2\right.$, and $\left.\ell_{B}=1 / \epsilon_{1}\right)$. The force is obtained in an exact way using the ICMMM2D method (dotted line) and with ICCMMM2D (solid line). The inset shows the relative error between the exact ICMMM2D calculation and three approximate methods, namely, ICCMMM2D (solid line), ICCELC (dashed line), and ICCP3M (dot-dashed line).

only on the results obtained via using the accuracy for the various electrostatics solvers provided by optimal tuning implemented in ESPResSo. ${ }^{51}$ In the case of charges in front of a planar dielectric interface, the exact solution in 2D periodicity can be obtained employing the ICMMM2D method, while for other simple geometries, like a charge in a cylinder or in front of a sphere, a big simulation box can be exploited to minimize the effect of periodic boundary conditions, allowing to compare $\mathrm{ICC}^{\star}$ calculations to known analytical results.

\section{Charges near planar interfaces and in a slit}

The first and the simplest test case that can be set up for the $\mathrm{ICC}^{\star}$ method consists in measuring the force acting on a single charge located in front of a planar dielectric interface, due to the presence of induced charges. The natural choice for the periodicity of this system is the two-dimensional (2D) one. In this case, not just a single particle but an infinite 2D lattice is simulated. As a consequence, the (approximate) values of the force can be obtained by using the ICCMMM2D and ICCELC methods. These results can be compared with the exact ones provided by ICMMM2D. Although, strictly speaking, ICCP3M solves the problem in a different periodicity, we also checked its results in order to test the influence of 3D periodicity.

In Fig. 2, the comparison between the approximated and the exact solutions is reported. The system consists of a single charge $q$ in a box of $20 \ell \times 20 \ell \times 20 \ell(\ell$ is the length unit) and, a dielectric interface located at $z=0$ that separates two media of dielectric permittivity $\epsilon_{1}=4$ and $\epsilon_{1}=2$, respectively. The real charge is positioned at different distances from the interface in the medium of dielectric permittivity $\epsilon_{1}$. The strength of the electrostatic interaction has been set by choosing the Bjerrum length $\ell_{B}=q^{2} /\left(k_{B} T \epsilon_{1}\right)$ to be $\ell_{B}=\ell / \epsilon_{1}$, where $k_{B}$ is Boltzmann constant and $T$ is the temperature.

The accuracy goal for the absolute pairwise force in the underlying ELC and MMM2D algorithms has been set to 
$10^{-7}$. For distances larger than three lattice units, the $\mathrm{ICC}^{\star}$ calculations managed to approximate the exact solution with a relative accuracy of about $10^{-6}$, which is close to the limit set by the accuracy of MMM2D and ELC. Rather predictably, when the distance from the lattice becomes comparable with the lattice size itself, the deviation from the exact solution starts increasing. Nevertheless, already at a distance of 1.5 lattice sizes, both the ICCMMM2D and ICCELC solutions are characterized by a relative error of only $1 \%$. Regarding the ICCP3M solution, the contribution of the periodic images along the third direction turns out to be, as expected, quite relevant. Therefore, for this kind of problems it is advised to always choose a solver which implements the correct periodicity, hence, MMM2D or ELC. For fully 3D problems, however, there is no problem in choosing $\mathrm{P} 3 \mathrm{M}$ as the correct electrostatic solver. It has to be noticed that, in the case of highly inhomogeneous systems, the sum of the squared charges $Q^{2}=\Sigma q_{i}^{2}$ might change considerably during the simulation run since the induced charge on a surface element is not a conserved quantity. Algorithms such as P3M or ELC will then need to be retuned when this change becomes substantial because their accuracy depends on $Q$. However, this situation is generally not encountered for equilibrium simulations involving a moderately high or high number of particles. Notice that the total charge is on the contrary conserved.

Given the results presented in Fig. 2, one could be tempted to consider ICCMMM2D and ICCELC to be equivalent choices for solving $2 \mathrm{D}$ problems. On the practical level though, one has also to take care about the algorithm performances. Since the $\mathrm{ICC}^{\star}$ methods inherit the scaling features from the underlying electrostatic algorithms, the performances of ICCMMM2D and ICCELC depend strongly on the system size. The computing time scales like $(N+M)^{5 / 3}$ and $(N+M) \log (N+M)$, respectively. This means that, already for a system with a few hundred induced charges, the ICCELC algorithm becomes noticeably faster than its MMM2D-based counterpart.

In order to test the method in the presence of multiple interfaces with different dielectric permittivity, we have computed the force acting on a charge placed in a dielectric slit of width $20 \ell$ and dielectric permittivity $\epsilon_{\mathrm{mid}}=4$, surrounded on the left and on the right by two regions of permittivity $\epsilon_{\text {left }}=2$ and $\epsilon_{\text {right }}=8$, respectively. We compared the force acting on the particle computed with ICCMMM2D and with ICMMM2D, showing the results in Fig. 3. In addition, we also show the differences in the ICCMMM2D force between this system and one characterized by $\epsilon_{\text {left }}=\epsilon_{\text {right }}=2$. The comparison shows that $\mathrm{ICC}^{\star}$ is able to compute correctly the forces in presence of multiple dielectrics, since it is able to resolve even the tiny differences (which ranges from $0.001 \%$ to $1 \%$ ) between the two systems, although the method accuracy is high enough only when the distance from the left interface becomes larger than 2.5 lattice units.

The checks presented so far confirm that the $\mathrm{ICC}^{\star}$ method is able to solve the electrostatic problem in presence of multiple dielectrics to a very good accuracy, taking into account the periodic nature of the systems. In principle, however, in order to solve such planar problems, the use of $\mathrm{ICC}^{\star}$

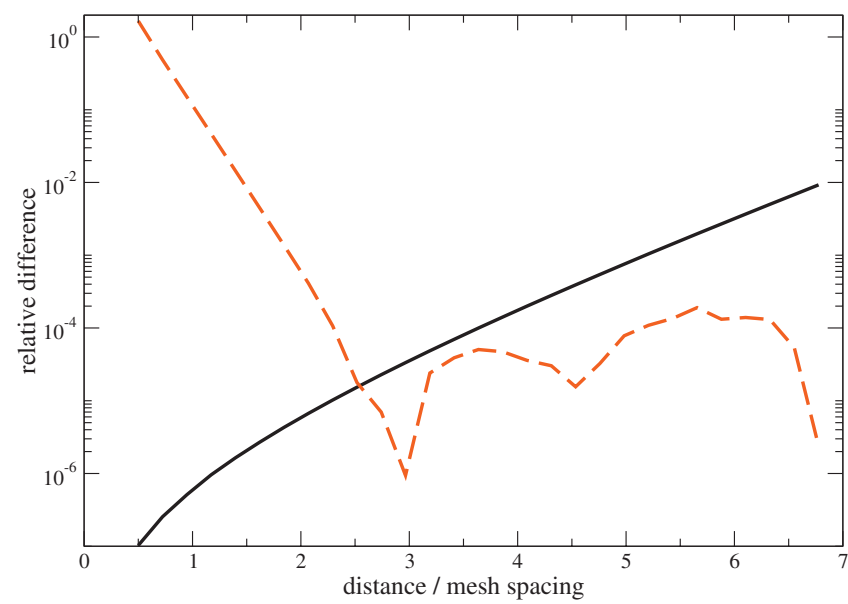

FIG. 3. Relative error (dashed line) between the ICCMMM2D and ICMMM2D solutions of the force acting on a charge in a dielectric slit, with three different (left, inner, and right) dielectric permittivities as a function of the distance from the left interface. The relative difference between the exact MMM2D solutions for the symmetric and asymmetric cases is also shown (solid line)

algorithms is not strictly required because one could use exact methods such as ICMMM2D or ELCIC. Indeed, the ICC ${ }^{\star}$ approach is best suited for interfaces of arbitrary shape, and the next checks are devoted to investigate the accuracy of the method in the case of two nonplanar geometries for which the analytic solution is known.

\section{A charge inside an infinite dielectric cylinder}

Here we consider a single particle located at the center of an infinite cylinder of radius $R$. The analytical result for this case is well known, ${ }^{38}$ and its Green function is a series of Bessel functions. The analytical expression for the induced surface charge density is given in cylindrical coordinates $(z, \rho, \phi)$ by

$$
\sigma(z, \phi)=\sum_{m=-\infty}^{\infty} \exp (i m \phi) \int_{0}^{\infty} d k A_{m}(k) \cos (k z) .
$$

The coefficients $A_{m}$ are defined as

$$
A_{m}=\frac{(\kappa-1)}{2 \pi^{2} R \epsilon_{0}} \frac{I_{m}(k d) K_{m}^{\prime}(k R)}{I_{m}^{\prime}(k R) K_{m}(k R)-\kappa I_{m}(k R) K_{m}^{\prime}(k R)},
$$

where $d$ is the distance between the charge and the cylinder center $\kappa=\epsilon_{2} / \epsilon_{1}$, and $I_{m}$ and $K_{m}$ are the Bessel and modified Bessel functions of the second kind, respectively, of the order $m$.

Of course, this solution is strictly valid only in the absence of periodic boundary conditions of any kind. However, by choosing the Bjerrum length smaller than the simulation box size and, in turn, a simulation box whose size is much larger than the cylinder radius, it is possible to minimize the effects of the imposed periodicity in the simulated system and to check the results of the method against the analytical ones. In particular, we have chosen to place a cylinder of radius $R=1.0 \ell$ in the center of a cubic simulation box whose edge is $10.0 \ell$ long. The ICCP3M method has been used to compute the electrostatic interaction for this system, using an induced charge mesh of $64 \times 64$ charges. 


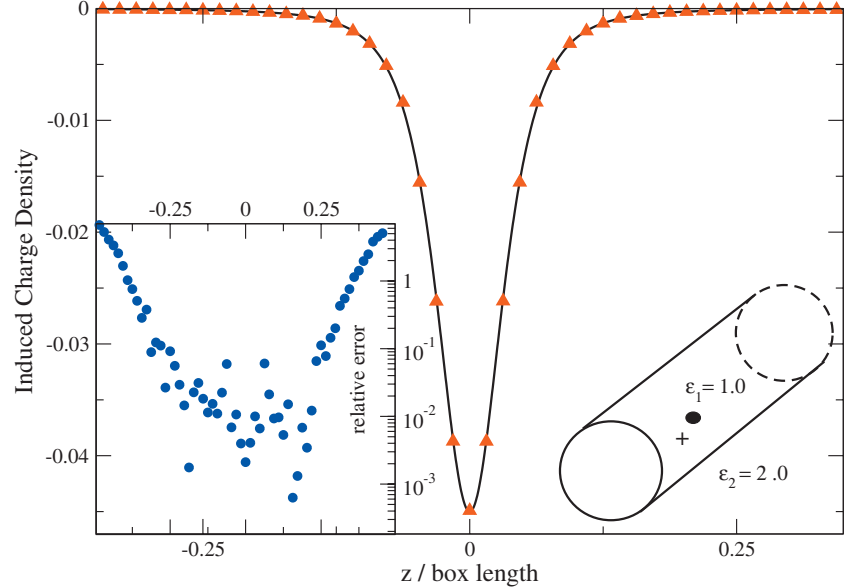

FIG. 4. Surface charge induced by a single unit charge located at the middle point $(z=0)$ on the axis of a dielectric cylinder, as a function of $z$. The relative error between the ICCP3M result (triangles) and the analytical formula (solid line) is presented in the inset. Due to the boundary condition in all three directions, the problem addressed by ICCP3M is actually that of infinitely many parallel cylinders.

The results of the simulation, together with the analytical ones, are plotted in Fig. 4. Here the inner and outer dielectric constants are set to $\epsilon_{1}=1$ and $\epsilon_{2}=2$, respectively. The real charge is located in the middle of the simulation box at $z$ $=0$, and the induced charge on the cylinder is computed as a function of the position $z$ along the cylinder axis. In the inset of Fig. 4, the relative error is also shown. Taking into account the fact that the contributions coming from the periodic images are not completely vanishing, the results of the method can be considered to be quite satisfactory. In the regions far from the real charge, high relative errors are observed. However, since the induced charge in those regions is extremely small, the error introduced in the calculation of the force is negligible. It is interesting to note that the combined error stemming from the periodicity of the system and from the discretization of the surface charge is of the same order of magnitude as the error introduced when simulating a finite, instead of periodic, system having the same geometrical characteristics, as it will be shown in Sec. IV B.

\section{A charge near a dielectric sphere}

Here we consider a point charge at distance $d$ from the center of a dielectric sphere. If spherical coordinates $(r, \theta, \phi)$ are used and the charge is placed along the $\theta=0$ axis, the polarization surface charge density may be expanded in terms of spherical harmonics, as a function of the polar angle $\theta,{ }^{38}$ namely,

$$
\sigma(\theta)=\sum_{l=0}^{\infty} A_{l} P_{l} \cos (\theta) .
$$

The coefficients $A_{l}$ are given by

$$
A_{l}=\frac{(1-\kappa) q R^{l-1} l(2 l+1)}{4 \pi \epsilon_{0} d^{l+1}[l(1+\kappa)+1]},
$$

where $\kappa$ is the ratio of dielectric constants and $R$ is the radius of the sphere. We have performed an ICCP3M calculation for a sphere of radius $R=\ell$ in a cubic box with an edge of

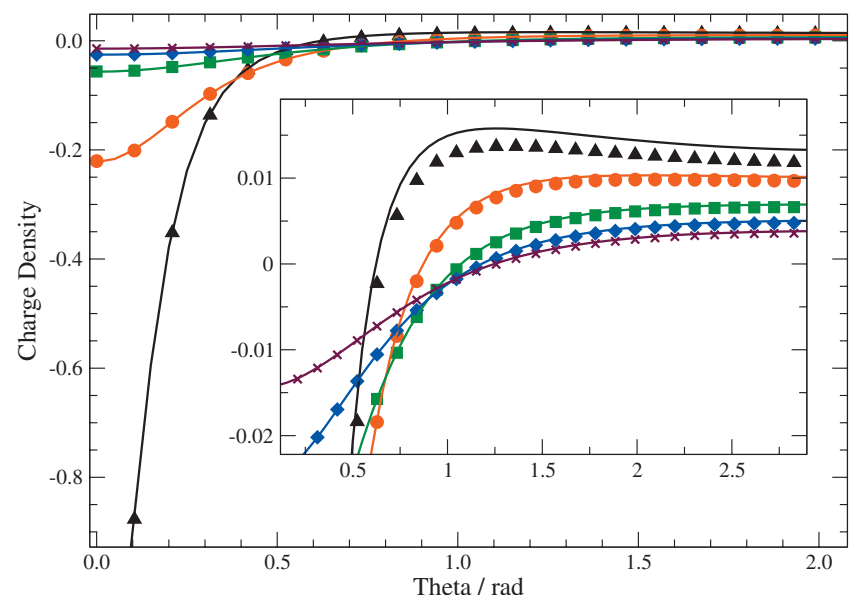

FIG. 5. Polarization charge induced on a dielectric sphere from a single charge placed in front of it, as a function of the polar angle $\theta$. Different symbols correspond to the ICCP3M estimate of the induced charge for a charge placed at 1.2 (triangles), 1.5 (circles), 2.0 (squares), 2.5 (diamonds), and $3.0 \ell$ (crosses), respectively, from the sphere center. The solid curves represent the corresponding analytical results, and the inset shows in detail the data in the charge density range $(-0.05$ and 0.015$)$ to better appreciate the agreement of the numerical calculation with the theory.

$20 \ell$, employing 900 surface elements. The dielectric permittivity of the sphere and of the surrounding medium are set to $\epsilon_{2}=2$ and $\epsilon_{1}=1$, respectively. The plots in Fig. 5 report the induced charge density on the sphere surface, as a function of $\theta$, for different distances of the real charge from the sphere surface. The results of the ICC ${ }^{\star}$ method, compared to the analytical ones (also reported in Fig. 5), are particularly good even in this geometric case where no direction on the interface has zero curvature. As expected, the deviations from the analytical result are more pronounced when the real charge is located close to the induced charges mesh, as it can be clearly seen in the inset of Fig. 5.

\section{Salt solution confined in a slit pore}

The test cases show so far involved only one charge in front of a dielectric interface. To show how $\mathrm{ICC}^{\star}$ behaves in a more realistic scenario, we set up a Langevin dynamics simulation of a salt solution confined to a slit pore. The solvent (middle region) is characterized by a Bjerrum length $\ell_{B}=\ell$ and a dielectric permittivity $\epsilon_{\mathrm{mid}}=1$. The dielectric permittivity of the left and right walls have been set to $\epsilon_{\text {left }}$ $=80$ and $\epsilon_{\text {right }}=1$, respectively. This choice has been taken to show the differences between the presence and absence of dielectric mismatch. However, since a wall-even with no dielectric mismatch-generates a depletion layer, ${ }^{52}$ we decided to model the other region with a dielectric permittivity higher than the solvent one to produce an opposite, more evident, effect of attraction to the wall. $N_{\text {salt }}=N_{+}+N_{-}$ monovalent salt ions, with $N_{-}=N_{+}=50$ were introduced in a simulation box of size $10 \ell \times 10 \ell \times 5 \ell$, and confined along the $z$ axis employing soft walls interacting with every particle via truncated Lennard-Jones interaction potential

$$
U_{\mathrm{LJ}}(z)=4 \varepsilon_{\mathrm{LJ}}\left(\frac{z}{\sigma}\right)^{12}-\left(\frac{z}{\sigma}\right)^{6}+\varepsilon_{\mathrm{LJ}} \quad z<2^{1 / 6} \sigma,
$$




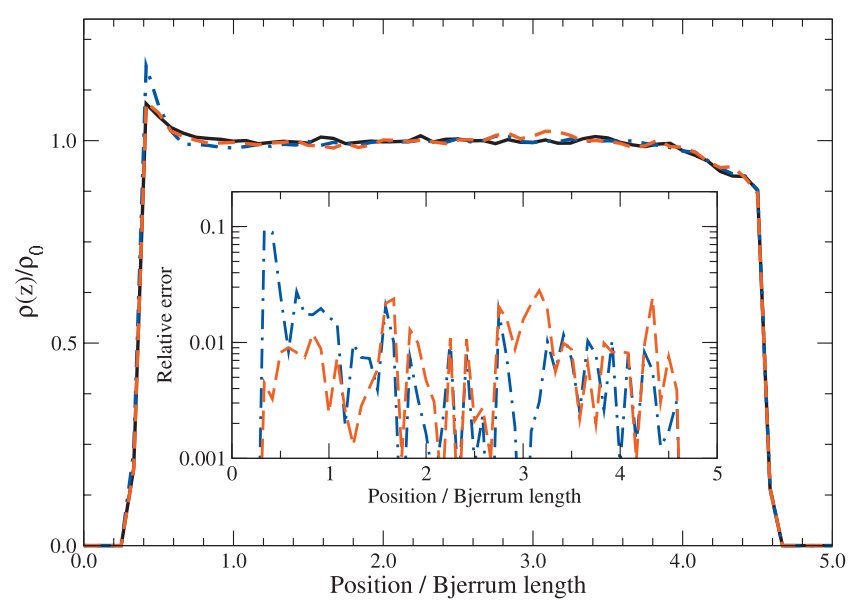

FIG. 6. The density profile $\rho(z)$ (normalized to the bulk density $\rho_{0}$ ) of the salt solution confined in a slit pore. The dielectric permittivity in the middle region is $\epsilon_{\text {mid }}=1$ like in the right region. The left region is characterized by a dielectric permittivity $\epsilon_{\text {left }}=80$. The three curves are relative to the exact ICMMM2D results (black, solid line), and to the ICCMMM2D results with a $5 \times 5$ (blue, dot-dashed line) and $10 \times 10$ mesh (red, dashed line). The position along the channel is expressed in units of the Bjerrum length $\ell_{B}$ $=\ell$. The inset shows the relative difference of the two ICCMMM2D simulation results $(5 \times 5$ mesh, blue dot-dashed line; $10 \times 10$ mesh, red dashed line) with the ICMMM2D result.

$$
U_{\mathrm{LJ}}(z)=0 \quad \text { otherwise }
$$

where $z$ is the distance of a particle from the wall, $\varepsilon_{\mathrm{LJ}}=k_{B} T$, and $\sigma=0.4 \ell$. Ions are interacting through the same truncated Lennard-Jones interaction and Coulomb potential, computed via the MMM2D algorithm with a precision of $10^{-3}$. Salt ions have been initially placed at random positions, taking care to avoid strong overlap between them.

The Langevin equations of motion have been integrated using a velocity Verlet algorithm at a reduced temperature equal to 1 . After an equilibration phase of $50 \times 10^{3}$ integration steps (with time-step $\Delta t=0.001$ ), equilibrium configurations were sampled every 100 integration steps. The resulting density profile has been reported in Fig. 6 , using $5 \times 5$ and $10 \times 10 \mathrm{ICC}^{\star}$ meshes, as well as the result of the same simulation performed using ICMMM2D instead of ICCMMM2D. Since the Hamiltonian of the system is invariant under charge reversal, the charge density profile of negative and positive ions should and did coincide within statistical errors.

From the data reported in Fig. 6 it is evident that a 10 $\times 10$ mesh is fine enough to reproduce a density profile in agreement with the exact result of ICMMM2D within a relative error of $3 \%$. The effect of the dielectric mismatch is quite striking, as it compensates and reverts the effect of depletion introduced by the presence of the wall (clearly seen at the right boundary, where the dielectric mismatch is 0 ). The artifacts introduced by a poor discretization can be seen from the data corresponding to a $5 \times 5 \mathrm{ICC}^{\star}$ mesh. In this case, an excess density is found at the left boundary, and correlations develop further until the middle of the box is reached. The effectiveness of the $10 \times 10$ grid is most probably related to the fact that such a mesh size is not greater than the Bjerrum length, and is therefore capable to represent well enough the theoretical, uniform polarization charge density.
The simulations were run for $70 \mathrm{CPU}$ hours on a cluster of modern personal computers and completed about $7.3 \times 10^{6}, 3.4 \times 10^{6}$, and $4.5 \times 10^{6}$ integration steps for the $5 \times 5,10 \times 10$, and ICMMM2D cases, respectively. In the $10 \times 10$ mesh case, which reproduced the correct results for the ion distribution, ICCMMM2D performed slightly worse $(\simeq 25 \%)$ than ICMMM2D. However, the small performance loss is, to our opinion, largely counterbalanced by the ability of $\mathrm{ICC}^{\star}$ to simulate nonplanar interfaces.

\section{B. Comparison to ICC}

In this section we compare the characteristics of $\mathrm{ICC}^{\star}$ to those of ICC, one of the alternative methods to compute the electrostatic interaction in presence of dielectric interfaces which has been briefly described in Sec. III. From the point of view of the variety of problems that can be addressed, the principal difference between the two approaches is the ability of $\mathrm{ICC}^{\star}$ to automatically take into account the periodicity of the system, according to the underlying algorithm employed to compute the bulk electrostatic interactions. This allows to describe infinite systems and to compute the interactions consistently when periodic boundary conditions are present. This is the most frequently occurring case in classical softcondensed matter simulations. In the case of ICC, on the contrary, only finite systems can be described. Moreover, in $\mathrm{ICC}^{\star}$ the case of many surfaces with distinct dielectric permittivity can be described naturally.

From the point of view of resources requirement and computational cost, the two approaches are quite different. The memory requirements of ICC are dramatically larger in comparison to those of $\mathrm{ICC}^{\star}$ since the occupation of the matrix $A$, which contains the geometrical information of the interface, scales like the square of the number of boundary elements. In the $\mathrm{ICC}^{\star}$ case, on the contrary, the memory requirements scale linearly with the number of boundary elements.

Regarding the performances, ICC has the advantage that the matrix $A$ has to be constructed only once at the beginning of the simulation. Still, the matrix has to be applied to the vector $\mathbf{b}$, in order to assign the polarization charges. The multiplication involves approximately $\mathcal{O}\left(M^{2}\right)$ operations while ICCP3M scales as $(N+M) \log (N+M)$. Nevertheless, the efficiency of the algorithms has to be checked explicitly in order to assess the importance of the scaling prefactors in the range of parameters that characterize a realistic simulation. In order to do so, we compared the computing times of ICC and ICCP3M for the problem of the charge in the cylinder. Here we implemented exactly the algorithm proposed by Boda and co-workers ${ }^{25}$ for comparison. The respective accuracy of the algorithms and the combined effect of errors introduced by nearest neighbor interaction (ICC) or periodic boundary conditions (ICCP3M) are summarized in Table I. There, the relative deviation from the analytical result for the charge density at $z=0$ is shown for different mesh sizes. In order to reach an equivalent accuracy, a double sized mesh has to be employed in the ICC algorithm.

The CPU time needed to evaluate the electrostatic interactions using a $64 \times 64$ mesh for both ICC and ICCP3M 
TABLE I. Differences between the induced charge density at the maximum of the distribution between ICC (various induced charges mesh size), ICCP3M (for an induced charges mesh size of $64 \times 64$ ), and the analytical solution for the infinite cylinder.

\begin{tabular}{lcc}
\hline \hline Method & Mesh size & Relative error \\
\hline ICC & $100 \times 100$ & 0.0020 \\
ICC & $80 \times 80$ & 0.0025 \\
ICC & $64 \times 64$ & 0.0031 \\
ICC & $32 \times 32$ & 0.0074 \\
ICC & $16 \times 16$ & 0.0283 \\
ICCP3M & $64 \times 64$ & 0.0021 \\
\hline \hline
\end{tabular}

methods are shown in Fig. 7. The measured CPU time clearly shows that besides the better scaling behavior, ICCP3M is also characterized by more favorable prefactors which lead to overall better performances in comparison to ICC.

It has to be noticed, however, that the ICCP3M calculations have been done starting from a randomly distributed surface charge. In a real-case MD simulation, after the induced charge distribution for the initial MD step has been computed, a lower number of iterations will probably be required in order to converge since the induced charge distribution is not expected to change dramatically after a small displacement of the real charges. Usually, results converge in one to three iterations. In order to quantify this, we have computed the average number of iterations which are needed to reach convergence $\left(\Delta=10^{-4}\right)$ for the problem of a charge in a dielectric slit consisting of three different dielectric regions. The charge has been placed at ten different positions and, for each of those, the ICCP3M algorithm has been applied employing a randomly induced charge distribution as a starting guess. Subsequently, the charge has been shifted by a tiny amount $(0.03 \ell$, a plausible displacement in a typical MD step) and the induced charge distribution has been recalcu-

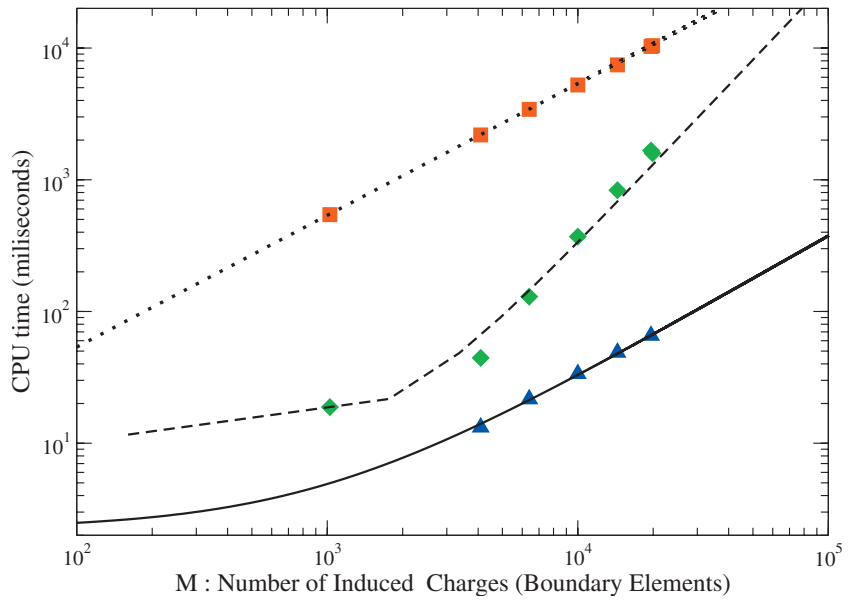

FIG. 7. Performance comparison between $\mathrm{ICC}$ and $\mathrm{ICC}^{\star}$ for the problem of a charge in a dielectric cylinder. The squares represent the time needed by ICC to compute matrix the $A$, while the diamonds represent the time spent in multiplying matrix $A$ with vector $c$. In comparison, the triangles represent the computing time needed by ICCP3M to reach convergence $\left(\Delta=10^{-4}\right)$. The dotted, dashed, and solid lines represent fits of the $M^{2}, \log (M+$ const $)$ $+M^{2}$, and $(M+N) \log (M+N)$ scalings, respectively.

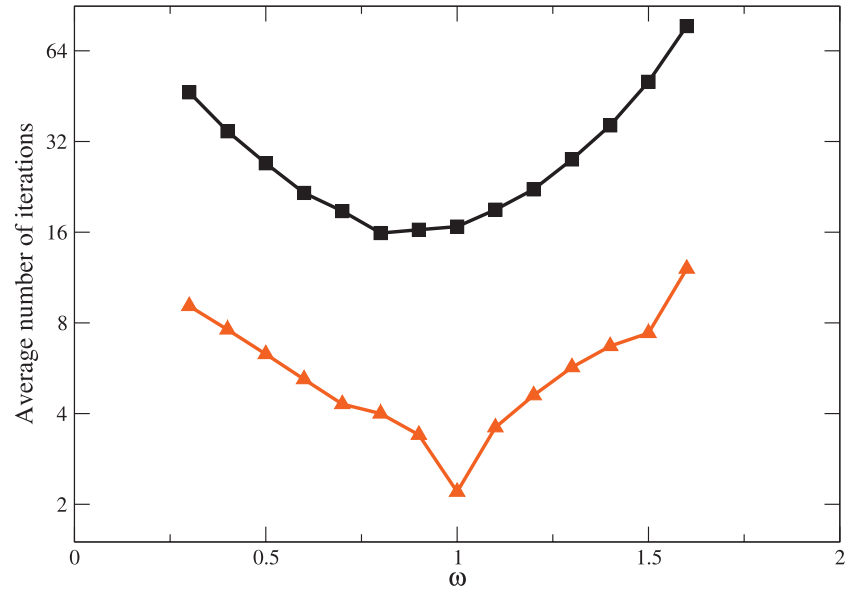

FIG. 8. Number of iterations needed to reach convergence $\left(\Delta=10^{-4}\right)$ as a function of the relaxation parameter $\omega$, for the case of a single charge in the dielectric slit (averaged over ten positions of the real charge). For every point ICCMMM2D has been applied first with random initial conditions (squares) and subsequently, after a small displacement of the charge (triangles), using the previous value of the induced charges as a starting distribution.

lated, this time using as initial guess for the induced charge distribution the values obtained from the previous iteration.

The results, averaged over ten starting positions, are presented in Fig. 8 in logarithmic scale. In the case of a random initial distribution, the number of iterations is minimized by choosing a relaxation parameter from the interval $(0.8,1)$ but still remains high. In the other case, the best relaxation parameter is $\omega=1$ which provides, on average, convergence in about two steps. This fast convergence shows how well in fact the method is suitable for being employed in any MD simulation scheme. Note, that the latter results are strictly related to the characteristics of the simulated system. In other words, the value of the optimal relaxation parameter is not universal and has probably to be redetermined when simulating different systems. Alternatively, an adaptive method (see, for example, Ref. 19) could be also implemented.

\section{CONCLUSIONS}

In this paper we put forward the combination of boundary element method and different families of electrostatics solvers to compute fast and efficiently the electrostatic interaction for systems obeying one-dimensional, 2D, and 3D periodic boundary conditions in presence of sharp and static dielectric interfaces. The interfaces can be arbitrary in number, shape, and value of their dielectric permittivity. The effect of dielectric media polarization is taken into account by solving an equivalent electrostatic problem formulated in terms of surface induced charges and solved using an iterative approach. The validity of the technique has been checked against different test cases, and both analytical and exact numerical results agree very well with the $\mathrm{ICC}^{\star}$ ones for planar (single or multiple), cylindrical, and spherical dielectric interface. Moreover, the algorithm converges in a limited number of iterations (one to three). The overhead that is introduced by treating dielectric interfaces using $\mathrm{ICC}^{\star}$ is therefore small, and allows to employ the method also in large-scale coarse-grained simulations. The $\mathrm{ICC}^{\star}$ algorithms 
scale basically like the employed electrostatic solvers and can therefore reach linear-scaling behavior for the cases of mesh methods, multigrid algorithm, or FMM approaches. The presented ICC ${ }^{\star}$ class of algorithms has been implemented in the ESPResSo (Ref. 51) MD simulation package.

\section{ACKNOWLEDGMENTS}

The authors thank K. Grass and S. Kesselheim for helpful discussions about the $\mathrm{ICC}^{\star}$ method and its implementation. Financial support by the Volkswagen Foundation, The BMBF, the DAAD, and the Alexander von Humboldt Foundation are gratefully acknowledged, as well as the use of the compute cluster of the Center for Scientific Computing (CSC) at Goethe University Frankfurt/Main, and the Wiglaf computer cluster at the Department of Physics of the University of Trento.

${ }^{1}$ S. J. Marrink, A. H. de Vries, and A. E. Mark, J. Phys. Chem. B 108, 750 (2004).

${ }^{2}$ S. J. Marrink, H. J. Risselada, S. Yefimov, D. P. Tieleman, and A. H. de Vries, J. Phys. Chem. B 111, 7812 (2007).

${ }^{3}$ M. Brodholt, J. Sampoli, and R. Vallauri, Mol Phys. 85, 149 (1995).

${ }^{4}$ G. Lamoureux, E. Harder, I. V. Vorobyov, B. Roux, and A. D. MacKerell, Jr., Chem. Phys. Lett. 418, 245 (2006).

${ }^{5}$ T. A. Halgren and W. Damm, Curr. Opin. Struct. Biol. 11, 236 (2001).

${ }^{6}$ J. Warwicker and H. Watson, J. Mol. Biol. 157, 671 (1982).

${ }^{7}$ M. Davis and J. McCammon, J. Comput. Chem. 12, 909 (1991).

${ }^{8}$ S. Chung, T. Allen, M. Hoyles, and S. Kuyucak, Biophys. J. 77, 2517 (1999).

${ }^{9}$ D. Levitt, Biophys. J. 22, 209 (1978).

${ }^{10}$ B. Honig, K. Sharp, and A. Yang, J. Phys. Chem. 97, 1101 (1993).

${ }^{11}$ B. Lu, X. Cheng, J. Huang, and J. A. McCammon, Proc. Natl. Acad. Sci. U.S.A. 103, 19314 (2006).

${ }^{12}$ M. D. Altman, J. P. Bardhan, J. K. White, and B. Tidor, J. Comput. Chem. 30, 132 (2009).

${ }^{13}$ T. Brumleve and R. Buck, J. Electroanal. Chem. 90, 1 (1978).

${ }^{14}$ D. Chen, J. Lear, and B. Eisenberg, Biophys. J. 72, 97 (1997).

${ }^{15}$ B. Corry, S. Kuyucak, and S. Chung, Biophys. J. 78, 2364 (2000).

${ }^{16}$ S. Rush, A. Turner, and A. Cherin, J. Appl. Phys. 37, 2211 (1966).

${ }^{17}$ S. Miertuš, E. Scrocco, and J. Tomasi, Chem. Phys. 55, 117 (1981).

${ }^{18}$ P. B. Shaw, Phys. Rev. A 32, 2476 (1985).

${ }^{19}$ R. Bharadwaj, A. Windemuth, S. Sridharan, B. Honig, and A. Nicholls, J.
Comput. Chem. 16, 898 (1995).

${ }^{20}$ J. Liang and S. Subramaniam, Biophys. J. 73, 1830 (1997).

${ }^{21}$ D. Chipman, J. Chem. Phys. 120, 5566 (2004).

${ }^{22}$ K. Sharp and B. Honig, Annu. Rev. Biophys. Biophys. Chem. 19, 301 (1990).

${ }^{23}$ J. Tomasi and M. Persico, Chem. Rev. (Washington, D.C.) 94, 2027 (1994).

${ }^{24}$ C. Cramer and D. Truhlar, Chem. Rev. (Washington, D.C.) 99, 2161 (1999).

${ }^{25}$ D. Boda, D. Gillespie, W. Nonner, D. Henderson, and B. Eisenberg, Phys. Rev. E 69, 046702 (2004).

${ }^{26}$ R. Allen, J. P. Hansen, and S. Melchionna, Phys. Chem. Chem. Phys. 3, 4177 (2001).

${ }^{27}$ J. Bardhan, J. Chem. Phys. 130, 094102 (2009).

${ }^{28}$ J. Bardhan, R. Eisenberg, and D. Gillespie, Phys. Rev. E 80, 011906 (2009).

${ }^{29}$ V. Rokhlin, J. Comput. Phys. 60, 187 (1985).

${ }^{30}$ M. Fenley, W. Olson, K. Chua, and A. Boschitsch, J. Comput. Chem. 17, 976 (1996).

${ }^{31}$ R. Zauhar and A. Varnek, J. Comput. Chem. 17, 864 (1996).

${ }^{32}$ Y. Vorobjev and H. Scheraga, J. Comput. Chem. 18, 569 (1997).

${ }^{33}$ E. Purisima, J. Comput. Chem. 19, 1494 (1998).

${ }^{34}$ A. Boschitsch, M. Fenley, and H. Zhous, J. Phys. Chem. B 106, 2741 (2002).

${ }^{35}$ A. Bordner and G. Huber, J. Comput. Chem. 24, 353 (2003).

${ }^{36}$ F. Figueirido, R. Levy, R. Zhou, and B. Berne, J. Chem. Phys. 106, 9835 (1997).

${ }^{37}$ E. Pollock and J. Glosli, Comput. Phys. Commun. 95, 93 (1996).

${ }^{38}$ J. D. Jackson, Classical Electrodynamics, 3rd ed. (Wiley, New York, 1999).

${ }^{39}$ R. Zauhar and R. Morgan, J. Mol. Biol. 186, 815 (1985).

${ }^{40}$ D. M. Young, Comput. Phys. Commun. 53, 1 (1989).

${ }^{41}$ L. Greengard and V. Rhoklin, J. Comput. Phys. 73, 325 (1987).

${ }^{42}$ R. W. Hockney and J. W. Eastwood, Computer Simulations Using Particles (McGraw-Hill, New York, 1981).

${ }^{43}$ M. Deserno and C. Holm, J. Chem. Phys. 109, 7678 (1998).

${ }^{44}$ M. Deserno and C. Holm, J. Chem. Phys. 109, 7694 (1998).

${ }^{45}$ A. Arnold, J. de Joannis, and C. Holm, J. Chem. Phys. 117, 2496 (2002).

${ }^{46}$ A. Arnold and C. Holm, Comput. Phys. Commun. 148, 327 (2002).

${ }^{47}$ A. Arnold and C. Holm, J. Chem. Phys. 123, 144103 (2005).

${ }^{48}$ A. Bródka, J. Chem. Phys. 125, 107103 (2006).

${ }^{49}$ S. Tyagi, A. Arnold, and C. Holm, J. Chem. Phys. 127, 154723 (2007).

${ }^{50}$ S. Tyagi, A. Arnold, and C. Holm, J. Chem. Phys. 129, 204102 (2008).

${ }^{51}$ H. J. Limbach, A. Arnold, B. A. Mann, and C. Holm, Comput. Phys. Commun. 174, 704 (2006)

${ }^{52}$ S. Cui and H. Cochran, J. Chem. Phys. 117, 5850 (2002). 